\title{
ANALYSIS OF DUAL BELL ROCKET NOZZLE USING COMPUTATIONAL FLUID DYNAMICS
}

\author{
Balaji Krushna.P' $^{1}$, P. SrinivasaRao ${ }^{2}$, B. Balakrishna ${ }^{3}$ \\ ${ }^{1}$ M.tech Student, ${ }^{3}$ Professor, Mechanical Engineering, UCEK-JNTUK, AP, India \\ ${ }^{2}$ Professor, Mechanical Engineering, Vardhaman College of Engg.., AP, India \\ balajikrushna.p@gmail.com,er.p.srinivas@gmail.com,balakrishnajntu06@gmail.com
}

\begin{abstract}
Concept of Altitude adaptive rocket nozzles recently received greater importance and interest in the space explorations and other such applications in space and rocket technology. The operations reliability of rocket launcher and the earth to orbit rocket launch are the crucial for the space transportation in the future. The performance of the engine components such as the power plant and the thrust delivery of the engine such as nozzles are in renovation for the greater performance and applicability for complex space applications. In the recent progress of the combustion expansion system the rocket nozzles are greatly revised from both application and design perspectives. One of such development is the dual bell nozzle. The publications indicate that the research on the concept of dual bell nozzle is tardy and there is no much progress from the inception of the idea. The specific application purpose designs are tested experimentally and implemented but the large scale development can only be possible if the generalized design parameters can be suggested. In the present paper one of such nozzle is selected and studied using computational fluid dynamics (CFD) and the results are synthesized for bench marking the general approach to study the Dual Bell nozzles.
\end{abstract}

The result shows the variation in the Mach number, pressure, temperature distribution and turbulence intensity.

Keywords: Altitude adaptation, Dual bell nozzle, Nozzle pressure ratio, Over-expansion factor.

\section{INTRODUCTION}

The most popular altitude-compensating rocket nozzle to date is the Dual bell nozzle, the origin of which dates back to Rocket dyne in the 1950s. This type of nozzle was designed to allow for better overall performance than conventional nozzle designs. This concept was thoroughly explored by the Germans during the time of the world war where in they were to be posted as a threat for their rivals. The structure of this type of nozzle roots on its advantages of minimizing the losses encountered in the previous versions of the conventional types. The literature survey has been thoroughly studied and their researches were beneficial.

A nozzle is used to give the direction to the gases coming out of the combustion chamber. Nozzle is a tube with variable to convert the thermo-chemical energy generated in the combustion chamber into kinetic energy. The nozzle converts the low velocity, high pressure, high temperature gas in the combustion chamber into high velocity gas of lower pressure and low temperature. Our study is carried using software's like Gambit 2.4 for designing of the nozzle and Fluent 6.3.2 for analyzing the flows in the nozzle.

In this paper CFD analysis of pressure and temperature for a dual bell rocket nozzle is analyzed with the help of fluent software. when the fuel and air enter in the combustion chamber according to the $\mathrm{x}$ and $\mathrm{y}$ plot, it is burning due to high velocity and temperature and then temperature increases rapidly in combustion chamber and convergent part of the nozzle and after that temperature decreases in the exit part of the nozzle.

\subsection{Need for New Nozzle Design}

The revolution in aerospace propulsion was increased greatly during World War 2. Faster, bigger and more efficient aerospace vehicles were required which led to the birth of Space research organizations like NASA. Speaking about the future, advanced rocket propulsion systems will require exhaust nozzles that perform efficiently over a wide range of ambient operating conditions. Most nozzles either lack this altitude compensating effect or they are extremely difficult to manufacture. Bell nozzles are currently used for all aerospace applications.

\section{METHODOLOGY}

A detailed study of concepts for the project is carried out. A procedural theoretical approach of nozzles is carried out and the models of nozzles namely the full length Dual-bell nozzle created using Fluent, Gambit software. The next step goes to 
the meshing and analysis of the dual bell model using FLUENT software. The behavior of flow along the dual bell nozzle is thus obtained and comparison on the basis of Mach number is henceforth done using theoretical calculations.

The material is selected as air and the density as ideal gas to make the solution simpler. Under the solve command the control is selected for limiting the pressure to a maximum of $5 e+7$ and Minimum of $1 e+4$. The initialization of value is computed from the inlet. It is also necessary to select the appropriate approximation required in the residual command under monitors and check in plot to visualize the progress of iteration. Once every parameter is described the iteration is performed till the value gets converged to required approximation. The Figures can be plotted between position in $\mathrm{x}$-axis and any other function in $\mathrm{y}$-axis from plot command or else to view vectors, contours or grid display command is to be chosen. A mathematical model consists of differential equations that govern the behavior of the physical system, and the associated boundary conditions.

\section{DUAL BELL NOZZLE}

The concept of nozzle was first studied at the Jet Propulsion Laboratory in 1949. In the late 1960s, Rocket dyne patented this nozzle concept, which has received attention in recent years in the U.S. and Europe. Figure illustrates the design of this nozzle concept with its typical inner base nozzle, the wall inflection, and the outer nozzle extension. This nozzle concept offers an altitude adaptation achieved only by nozzle wall inflection. In low altitudes, controlled and symmetrical flow separation occurs at this wall inflection Fig. 2 which results in a lower effective area ratio. For higher altitudes, the nozzle flow is attached to the wall until the exit plane, and the full geometrical area ratio is used. Because of the higher area ratio, an improved vacuum performance is achieved. However, additional performance losses are induced in dual-bell nozzles, as compared with two baseline nozzles having the same area ratio as the dual-bell nozzle at its wall inflection and in its exit plane.

Flow transition behavior in dual-bell nozzles strongly depends on the contour type of the nozzle extension. A sudden transition from sea-level to vacuum operation can be, at least theoretically, achieved by two different extensions, with a zero wall pressure gradient (constant pressure extension), or a positive wall pressure gradient (overturned extension). But a critical analysis of the transition behavior considering decreasing ambient pressures during the launcher ascent revealed that a considerable time with uncontrolled flow separation within the nozzle extension exists even for these types of extensions. The duration of this period can be reduced drastically by throttling the chamber pressure.

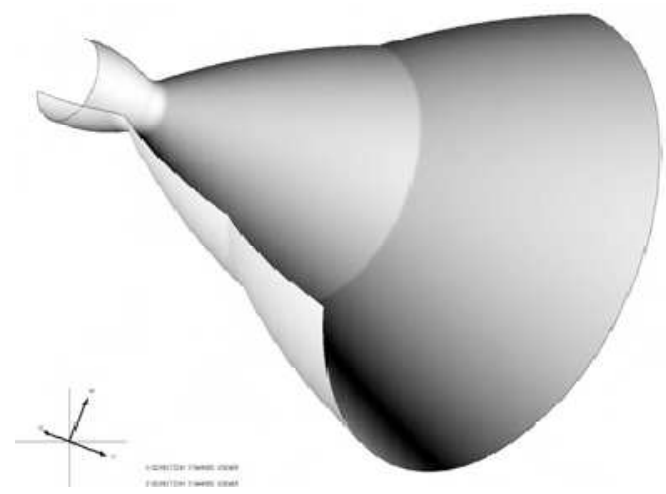

Fig .1 Sketch of a dual-bell nozzle

The main advantage of dual-bell nozzles as compared to other means of controlling nozzle flow separation is its simplicity because of the absence of any movable parts and, therefore, its high reliability. It is necessary to note that the external flow over the vehicle in flight reduces the pressure in the vehicle base region, where engines are installed. The ambient pressure triggering the flow transition is the vehicle base pressure instead of the atmospheric pressure at the specific flight altitude. As the base pressure is lower than the atmospheric pressure, the nozzle flow transition occurs at a lower altitude than the one showed in Fig.1 which slightly decreases the efficiency of the dual-bell nozzle operation along the trajectory. Despite the additional losses induced in dual-bell nozzles, they still provide a significant net impulse gain over the entire trajectory as compared to conventional bell nozzles. Independent combined launcher and trajectory analyses performed by Dasa within the European Space Agency Future European Space Transportation Investigations Program (ESA FESTIP) study and at DLR on SSTO vehicles powered with dual-bell nozzles result in a significant payload gain when compared with a reference launcher equipped with conventional nozzles.
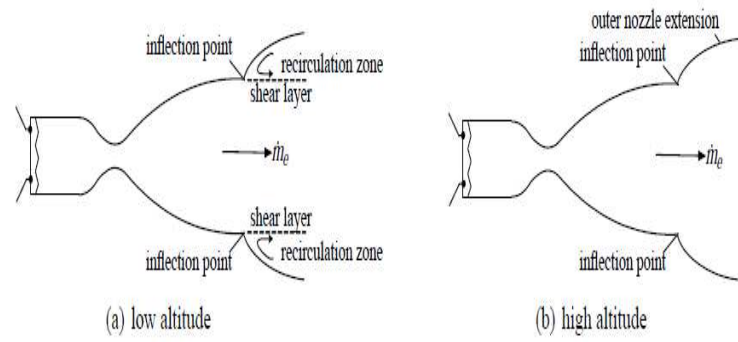

Dual-bell nozzle

Fig.2 Dual-bell nozzle (a) low altitude (b) high altitude 
Dual-bell nozzle designs accommodate several outlet pressures and provide a significant net impulse gain over the entire trajectory as compared to conventional bell nozzles. The concept proposes a typical inner base nozzle accompanied by an outer nozzle addition. The connecting point between radial contours is a wall inflection point. Figure 3(a) shows that the exhaust flow for lower altitude operation separates at the inflection point whereas Fig. 3(b) Shows that higher altitude flow remains attached until the exit plane of the outer nozzle extension.

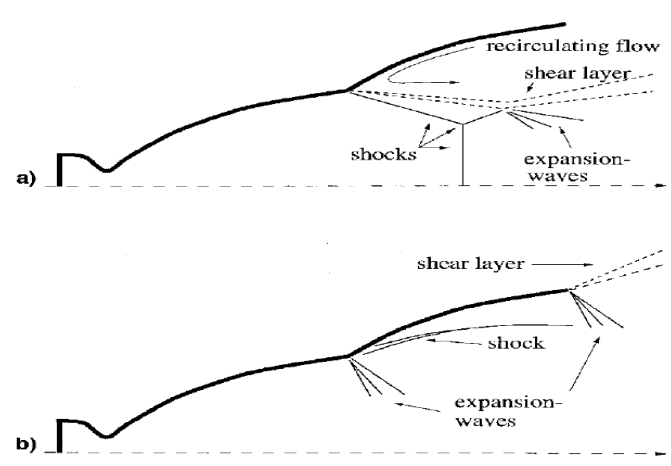

Fig 3 Flow field phenomena in dual-bell nozzles: a) sea-level mode with flow separation at the wall inflection point and $b$ ) altitude mode with a full- flowing nozzle.

For higher altitudes, the nozzle flow is attached to the wall until the exit plane, and the full geometrical area ratio is used. Because of the higher area ratio, an improved vacuum performance is achieved. However, additional performance losses are induced in dual-bell nozzles, as compared with two baseline nozzles having the same area ratio as the dual-bell nozzle at its wall inflection and in its exit plane. Below Figures illustrate the performance of a dual bell nozzle as a function of flight altitude in comparison with both baseline bell-type nozzles.

\subsection{Background:}

In supersonic flow, the influence of a small pressure disturbance is limited to a specific region.

$>\quad$ Pressure disturbance propagates relative to fluid as a spherical sound wave at local velocity of sound $a$.

$>\quad$ Center of sound wave moves downstream with velocity $u$.

$>$ Changes in fluid properties may be thought of as propagating along Mach lines:

$>\quad$ Mach line is straight, if flow upstream is uniform.

$>\quad$ All properties of flow immediately downstream of a Mach line are uniform.

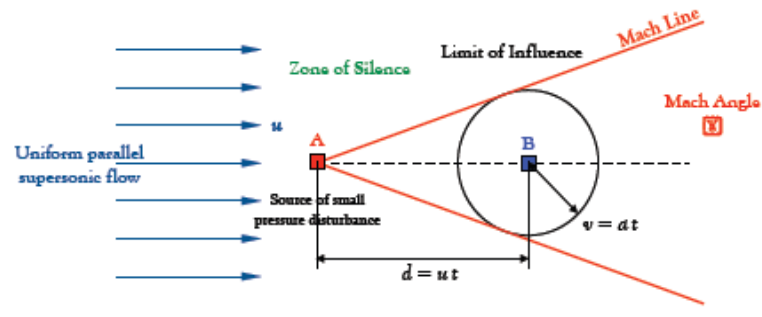

$$
\begin{aligned}
& \alpha=\sin ^{-1}\left(\frac{a t}{u t}\right)=\sin ^{-1}\left(\frac{1}{M}\right) \\
& \alpha=\tan ^{-1}\left[\frac{a t}{\sqrt{(u t)^{2}-(a t)^{2}}}\right]=\tan ^{-1}\left[\frac{1}{\sqrt{M^{2}-1}}\right]
\end{aligned}
$$

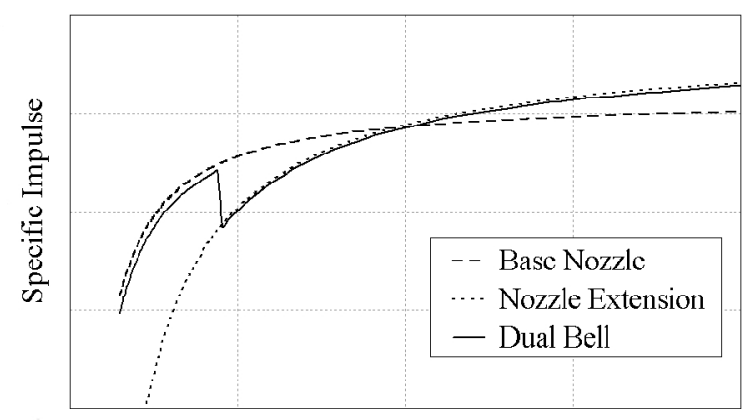

Flight Altitude

Fig 4 Performance data of a dual-bell nozzle

Performance is compared with two baseline bell-type nozzles as function of flight altitude (baseline nozzle 1: same area ratio as dual-bell base nozzle; baseline nozzle 2: same area ratio as nozzle extension)

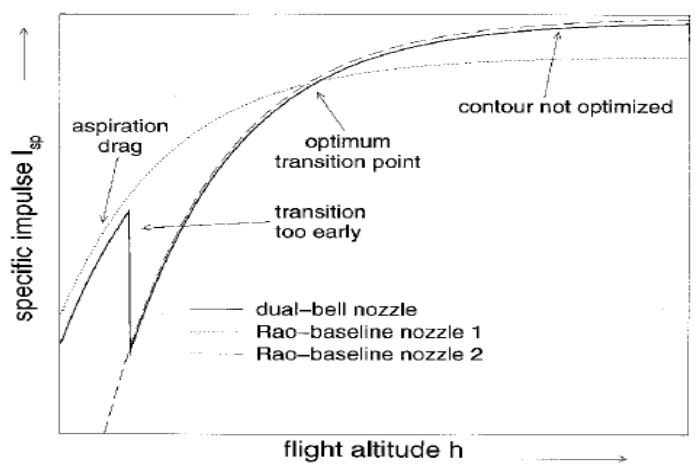

Fig 5 Performance characteristics of a dual-bell nozzle 
Performance is compared with two baseline bell-type nozzles as function of flight altitude (baseline nozzle 1: same area ratio as dual bell base nozzle; baseline nozzle 2: same area ratio as nozzle extension).

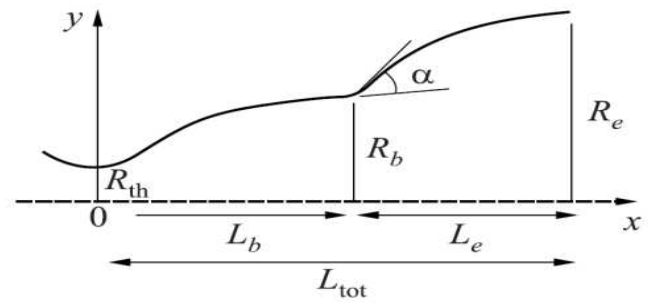

Fig.6.Geometrical parameters of a dual bell nozzle

\section{THEORETICAL APPROACH}

The theoretical approach can be identified as the coresection of the project whereas the efficiency analysisis carried out with design parameters. Rocket nozzle efficiency,

$\eta_{\mathrm{C}}=\frac{\text { Actual thrust coefficient }}{\text { Ideal thrust coefficient }}$

$\eta_{C}=\frac{\text { Cthrust.a }}{\text { Cthrust.i }}$

Actual Thrust Coefficient $=\frac{\text { Thrust }}{\mathrm{P}_{\mathrm{c}} \cdot \mathrm{A}_{\mathrm{t}}}$

Thrust force is given by,

$\mathrm{F}=\mathrm{A} * \mathrm{P}_{\mathrm{C}} \sqrt{\frac{2 \mathrm{~K}^{2}}{\mathrm{~K}-1}}\left(\frac{2}{\mathrm{~K}+1}\right)^{\frac{\mathrm{K}+1}{\mathrm{~K}-1}}\left[1-\left(\frac{\mathrm{P}_{\mathrm{e}}}{\mathrm{P}_{\mathrm{c}}}\right)^{\frac{\mathrm{K}-1}{\mathrm{~K}}}\right]+\left(\mathrm{P}_{\mathrm{e}}-\mathrm{P}_{\mathrm{a}}\right) \mathrm{A}_{\mathrm{e}}$

\section{DESIGN PARAMETERS}

\begin{tabular}{|l|l|}
\hline Design parameters & \multicolumn{1}{|c|}{ Values } \\
\hline Throat Raidus $\mathrm{R}_{\mathrm{th}}$ & $15 \mathrm{~mm}$ \\
\hline Exit area, $\mathrm{a}_{\mathrm{e}}$ & 14.3 in $^{2}$ \\
\hline Exit area ratio, $\mathrm{a}_{\mathrm{e}} / \mathrm{a}_{\mathrm{b}}$ & 4 \\
\hline Rocket flow specific heat ratio, $\gamma$ & 1.532 \\
\hline Nozzle exit mach number $\mathrm{m}_{\mathrm{e}}$ & 1.2 \\
\hline Rocket chamber pressure $\mathrm{p}_{\mathrm{c}}$ & $3200 \quad$ Psia \\
\hline Nozzle exit pressure $\mathrm{p}_{\mathrm{e}}$ & $3.41 \mathrm{e}+04$ pasc \\
\hline
\end{tabular}

\section{FLOW ANALYSIS}

The flow analysis for the dual bell nozzle is carried out using ANSYS 14.0 Fluent software. In this process first the models are imported, meshed and flow analysis is carried out in major three steps

1. The first step is GAMBIT, where the meshed model is imported and boundaries are created and corresponding boundary conditions are assigned to the boundaries.

2. The second step is FLUENT-SOLVER, where the solutions are obtained by solving the equations and process is highlighted in terms of codes and graphs and once the run is over it reaches next step.

3. The third step is FLUENT, where the corresponding contours are created for following major parameters such a Pressure, Temperature and Mach number.

\section{RESULTS AND DISCUSSION}

\subsection{Mach Number}

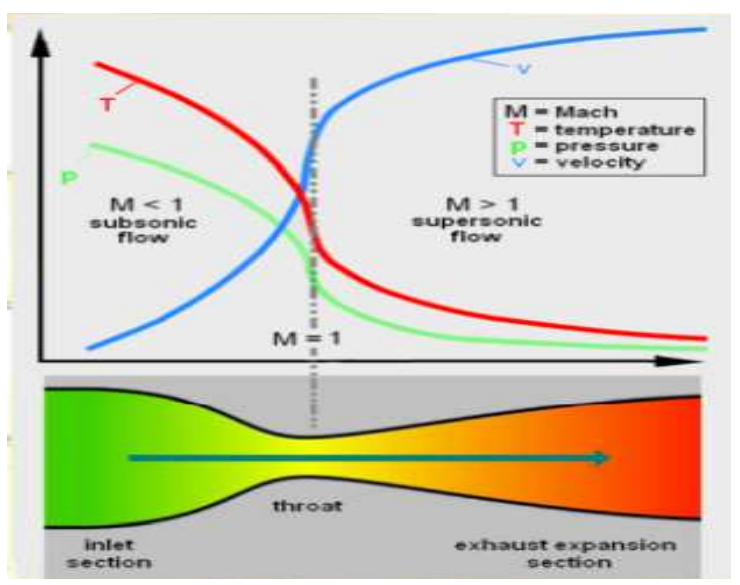

The velocity magnitude is found to increase as move from inlet to exit. The velocity at the inlet is $0.067 \mathrm{Mach}$ (subsonic). At the throat section the velocity varies from 0.06 Mach to 0.08 Mach. The velocity at the exit is found to be 1.2Mach (super-sonic)

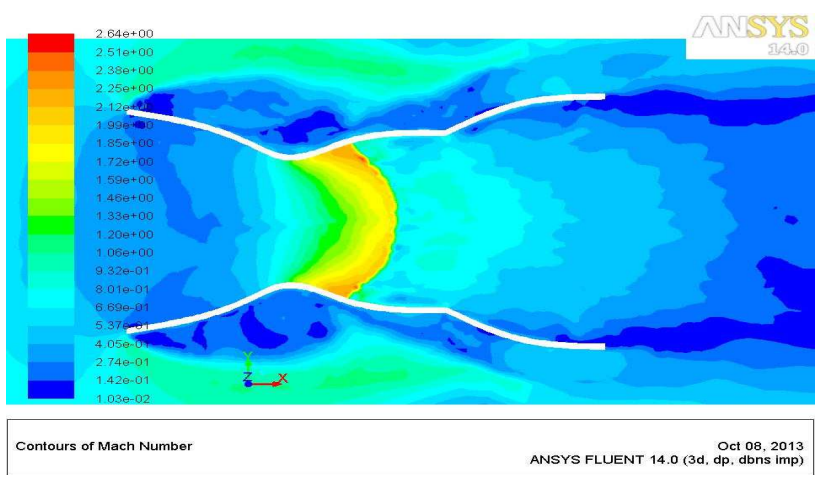

Fig.7 Contour of Mach number 


\subsection{Dynamic Pressure}

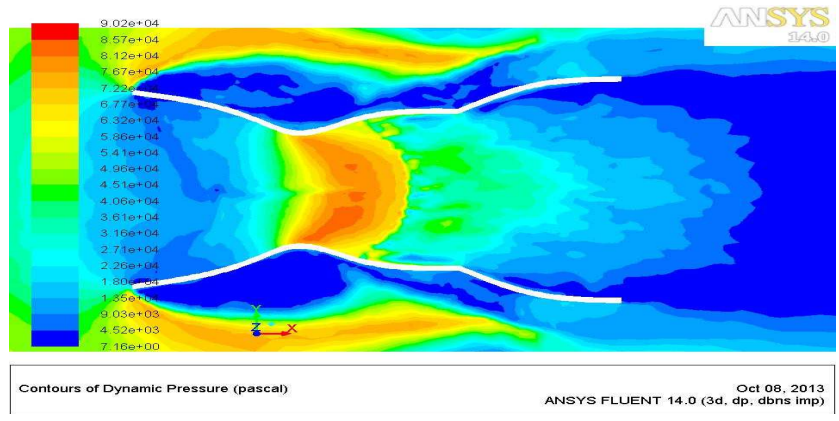

Fig.8 Dynamic Pressure (Pascal)

Dynamic pressure is the pressure that is exerted by a fluid. Specifically, it is the pressure measured when the fluid is still, or at rest. The above figure reveals the fact that the gas gets expanded in the nozzle exit. The Dynamic pressure in the inlet is observed to be $5.41 \mathrm{e}+04 \mathrm{~Pa}$ and as move towards the throat there is a increase and the value at the throat is found out to be $7.22 \mathrm{e}+04 \mathrm{~Pa}$. After the throat, there is a sudden increase in the Dynamic pressure at the axis which indicates the occurrence of the shock. After the shock there is a slight decrease in the pressure but it again rises at the second shock. Then it reduces to a value of $3.16 \mathrm{e}+04 \mathrm{~Pa}$ at the exit section due to the expansion of the fluid towards the exit of the nozzle.

\subsection{Static Pressure Graph}

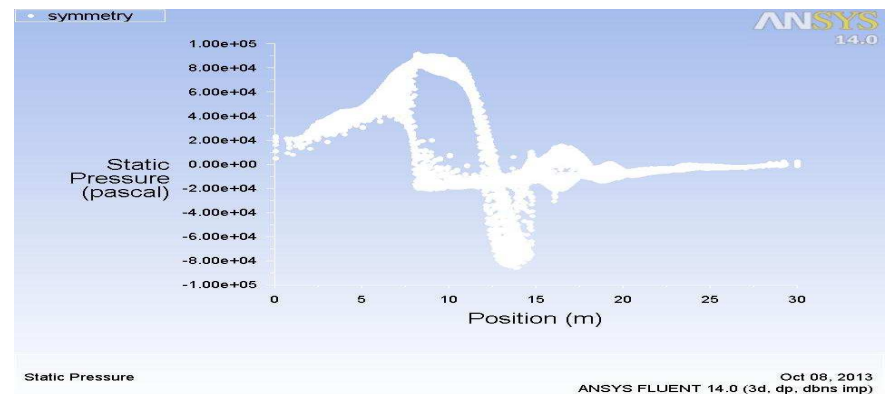

\subsection{Velocity}
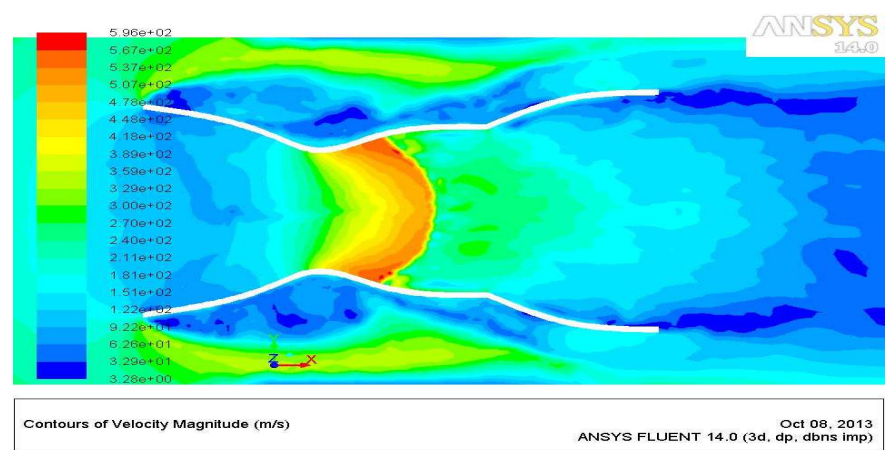

Fig.9 Contours of Velocity Magnitude (m/s)
The velocity magnitude is found to increase as move from inlet to exit. The velocity at the inlet is $1.22 \mathrm{e}+02 \mathrm{~m} / \mathrm{s}$. At the throat section the velocity varies from $2.40 \mathrm{e}+02 \mathrm{~m} / \mathrm{s}$ to 2.70 $\mathrm{e}+02 \mathrm{~m} / \mathrm{s}$. The velocity at the exit is found to be $3.29 \mathrm{e}+02$.

\subsection{Radial Velocity}

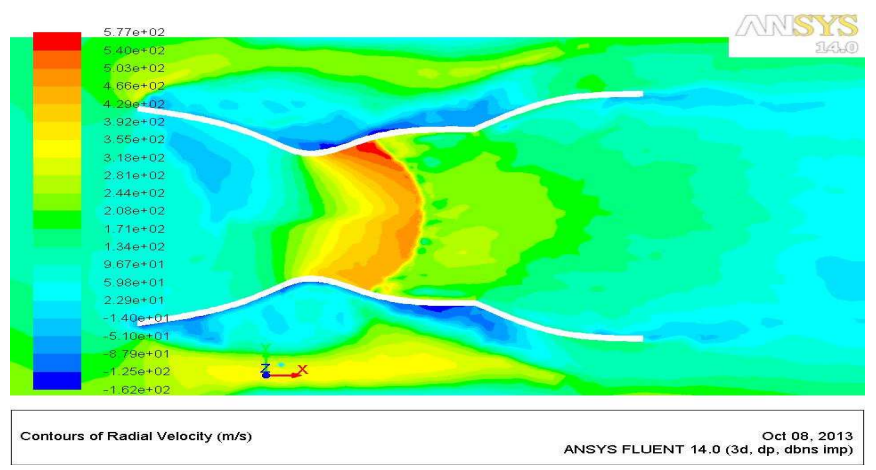

Fig.10 Contours of Radial Velocity $(\mathrm{m} / \mathrm{s})$

The Radial velocity magnitude is found to increase as move from inlet to exit. The velocity at the inlet is $2.29 \mathrm{e}+01 \mathrm{~m} / \mathrm{s}$. At the throat section the velocity varies from $1.34 \mathrm{e}+02 \mathrm{~m} / \mathrm{s}$ to $1.71 \mathrm{e}+02 \mathrm{~m} / \mathrm{s}$. The velocity at the exit is found to be 2.81 $\mathrm{e}+02 \mathrm{~m} / \mathrm{s}$.

\section{MERITS}

The dual bell nozzle has $90 \%$ overall better performance than the conventional bell-shaped nozzle. The efficiency at low altitudes is much higher because the atmospheric pressure restricts the expansion of the exhaust gas. A vehicle using a dual bell nozzle also saves $25-30 \%$ more fuel at low altitudes. At high altitudes, the dual bell nozzle is able to expand the engine exhaust to a larger effective nozzle area ratio. The dual bell design is suitable for Single Stage to Orbit (SSTO) flight. Other advantages are that the dual bell nozzle makes better use of the base area, and has higher thrust efficiency and thus a higher average specific impulse.

\section{CONCLUSIONS}

The following observations were found in the Dual bell nozzle

Mach number: The Mach number at the exit is found to be 1.2Mach (super-sonic).

Dynamic Pressure: The dynamic pressure value of 3.16 e+04 $\mathrm{Pa}$ at the exit section due to the expansion of the fluid towards the exit of the nozzle. Here pressure value is decreases inlet to exit area of nozzle.

Velocity: The velocity value of Nozzle increases inlet to exit the velocity at the exit is found to be 3.29 e+02. 
Radial Velocity: The Radial Velocity of bell nozzle increases from inlet to exit at the exit is found to be $2.81 \mathrm{e}+02 \mathrm{~m} / \mathrm{s}$.

\section{REFERENCES}

[1] Dumonov, G., Ponomaryov, N.B. and Voinov, A.L.,(1997) 'Dual-Bell Nozzles for Rocket Engines of Launch Vehicle Upper Stages and Orbital Transfer Vehicles,' $\quad$ AIAA Paper 97-3089, $33^{\text {rd }}$ AIAA/ASME/SAE/ASEE Joint Propulsion Conference \& Exhibit, USA, Luglio.

[2] Hagemann, G., Immich, H., Nguyen, T. V., and Dumnov, G. E.,(1998)“Advanced Rocket Nozzles,” Journal of Propulsion and Power, Vol. 14, No. 5, September-October pp. 620-634.

[3] Frey, M. and Hageman, G.,(1999) 'Critical Assesment of Dual-Bell Nozzles,' Journal of Propulsion and Power, Vol. 15, No.1, Jan.-Feb.

[4] Hagemann, G., Immich, H. and Preuss, A, ( 3-6 December 2002,)"Advanced Nozzle Concepts for Future Rocket EngineApplications', 4th International Conference on Launcher Technology, Liege, Belgium.

\section{BIOGRAPHIES}

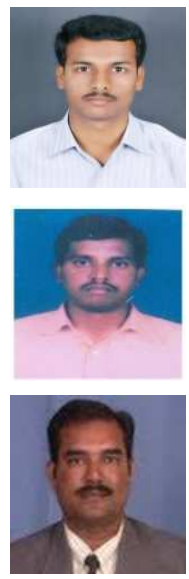

Balaji krushna .P M.tech (CAD/CAM) UCEKJNTU Kakinada

Dr. P. Srinivas rao Ph.D. Professor and Head, Dept of Aerospace Engineering Vardhaman College of Engineering Hyderabad

Dr. B. Balakrishna Ph.D. Professor in Mechanical Engineering \& Head, Dept. of Petroleum Engineering \& Petrochemical Engg, UCEK-JNTU Kakinada 